\title{
Estimates of male dispersal in Yponomeuta padellus (Lepidoptera: Yponomeutidae), the small ermine moth, by means of simulation
}

\author{
M. I. BROOKES* \& R. K. BUTLIN $\dagger$ \\ School of Pure and Applied Biology, University of Wales College of Cardiff, P.O. Box 915, Cardiff CF1 3TL, and \\ Department of Genetics and Biometry, University College London, 4 Stephenson Way, London NW1 $2 H E$, UK
}

\begin{abstract}
A model of male dispersal is described which simulates the observed recaptures from a series of mark-release-recapture experiments. The model aims to obtain an unbiased estimate of the dispersal parameter, $\sigma$, for males of Yponomeuta padellus (Lepidoptera: Yponomeutidae). Five parameters, each considered to be potentially important in an analysis of the dispersal patterns of $Y$. padellus, were incorporated into the model. Parameter values were varied individually in a stepwise fashion to create a large number of simulated data sets. The method of maximum likelihood was used to test the goodness of fit of each simulated data set against the observed distribution of recaptures to determine the optimal combination of parameter values. Using these same parameter values, the model was then modified by examining the movement of male moths in the absence of traps to obtain an estimate of $\sigma$. The 'best' estimate of $\sigma$ was found to be $2.9 \mathrm{~m} \mathrm{gen}^{-1 / 2}$, with an approximate upper confidence limit of $6.4 \mathrm{~m} \mathrm{gen}^{-1 / 2}$. The results suggest that dispersal in $Y$. padellus is extremely restricted relative to the area occupied by the total population and that $Y$. padellus is characterized by an extremely fine-scale population structure.
\end{abstract}

Keywords: gene flow, host races, likelihood, neighbourhood size, population structure, simulation.

\section{Introduction}

Genetic drift can have important effects on the geographical patterns of genetic variation in natural populations. In a continuous population, restricted dispersal will effectively prevent gene flow between individuals separated by distances greater than their dispersal range. If the effective density of individuals is sufficiently small, a single population may become genetically subdivided through genetic drift; this is the so called 'isolation by distance' model of population structure (Wright, 1943, 1951). The geographical scale of any gene frequency fluctuations produced by drift will depend on the dispersal rate, $\sigma$, the standard deviation of dispersal distances (along one axis of a bivariate distribution). $\sigma$ also determines the spatial scale over which these local fluctuations in gene frequencies will be correlated (Wright, 1951; Rohlf \& Schnell, 1971; Endler, 1979; Slatkin, 1985). The magnitude of these

*Present address and correspondence: Department of Genetics and Biometry, University College London, 4 Stephenson Way, London NW1 2HE, UK.

$\dagger$ Present address: Department of Genetics, The University, Leeds LS2 9JT, UK. fluctuations will depend on the neighbourhood size, $N_{b}$, defined as $4 \pi \sigma^{2} D$ where $D$ is the effective density of individuals.

In the summer of 1990 , a series of mark-releaserecapture experiments was carried out on a large, apparently continuous population of $Y$. padellus, as part of a direct investigation into the population structure of this species (Brookes \& Butlin, 1994). Y. padellus is associated with a variety of host plants, most commonly hawthorn, Crataegus monogyna, and blackthorn, Prunus spinosa. Significant gene frequency differences have been detected between some hostassociated populations, and it has been suggested that these populations are host races, and may represent the early stages of a sympatric speciation process (Menken, 1981, 1989; Menken et al., 1992). However, an estimate of the dispersal rate is required to predict the scale of genetic structuring which would be expected in a population of $Y$. padellus from purely random effects and which may account for apparent genetic differences between host-associated populations.

Accurately estimating the dispersal rate from trapping data is often complex. An observed distribution of recaptures represents a sample of the dispersing popu- 
lation, but mortality and fluctuating population size during dispersal, and the effect of the traps themselves, can all complicate the calculation of the dispersal rate directly from this observed distribution. Grouping spatial and temporal information on observed recaptures may help to simplify the approach but details in the data may be lost.

To estimate $\sigma$ for $Y$. padellus, a method was sought which made the most efficient use of all the information available in the recapture data and incorporated the effect of life-history patterns. Certain aspects of the biology of this species make it ideal for modelling dispersal in detail by means of computer simulation. Males are attracted to the pheromone of calling females and mating takes place on a host bush. The sites to which males disperse are therefore discrete rather than continuous. All the hawthorn and blackthorn bushes within the study population have been accurately mapped (over 600 in total). Thus, the exact positions of the sites to which males can potentially disperse are known. Females were shown to be largely sedentary, rarely dispersing away from the bush on which they were released (M. I. Brookes \& R. K. Butlin, unpublished data). Thus, the dispersal model is solely concerned with the movement of males.

The field experiments on male dispersal involved releasing marked males from three release bushes within the study population. Males were recaptured in traps baited with a single virgin female. Once a male was caught, it was effectively removed from the experiment. Fifteen traps were positioned on bushes surrounding each of the three release bushes, making 45 traps in total. For each grouping of 15 traps, one, two, four and eight traps were positioned on bushes at approximate radial distances of 2, 4, 8, 16 and $32 \mathrm{~m}$, respectively, from the release bush. Y. padellus is univoltine and the entire release experiment lasted a total of 24 days, coinciding with the period from initial emergence to decline of the wild moth population within the study site.

A model of male dispersal has been designed which simulates the observed distribution of recaptured marked male moths from the field experiments. Simulated data sets were compared with the observed recapture data using a likelihood analysis to optimize the values of each of five model parameters. Using these specific parameter values the model has then been modified by simulating the movement of moths in the absence of traps to obtain an estimate of the dispersal rate. As male dispersal appears to be intimately associated with the location of host bushes, simulations have been initiated at a variety of different bush locations within the study site to examine how variation in bush density might affect the dispersal rate.

\section{The model}

All programs were written in TURBO PASCAL, version 5.5 (TURBO PASCAL Reference Guide, 1988, Borland Int., Scotts Valley, CA). The main body of the program simulates the movement of an individual moth, starting from the specified co-ordinates of one of the three release bushes used in the 1990 field experiment. A specified number of inter bush movements are made on a 'daily' basis and continue up to a maximum of 24 days (the total duration of the 1990 field experiment), unless either the moth dies or it is caught in a trap. If the moth is caught, the trap number and the day of recapture are recorded by entering the event into the appropriate element of a $24 \times 45$ matrix of day against trap number, respectively. When a moth's movements are terminated, the model returns to the beginning of the main body of the program to simulate the movement of the next moth. For any particular combination of parameter values, the main body of the program is repeated 1000 times to simulate the dispersal of 1000 moths. At the end of a complete simulation (1000 moths), the final results take the form of a $24 \times 45$ matrix which displays the total number of moths caught in each trap on each day.

A list of the parameters incorporated into the model is given below. The words in italics refer to the names used to define each parameter as written in the computer program (available on request). For a further explanation of the model, refer to Fig. 1.

\section{Daily rate of mortality (mort)}

Movement parameter (gammafile). The bush to which a male moth moves at each movement event is determined by a variable drawn at random from a gamma distribution. A gamma distribution is described by two parameters, the 'shape' parameter $r$, and the 'scale' parameter $\lambda$ (Mood et al., 1974); $r$ remains constant and equal to 2.0 for all simulations; the movement parameter is therefore specified by $\lambda$. The moth moves to the bush whose distance from the current bush is closest to the value of the gamma variable. If the value of this chosen variable is less than half the distance to the nearest bush then, although a movement event is recorded, the moth remains on its original bush.

The choice of the gamma distribution with $r$ equal to 2.0 , as the most appropriate form of the movement parameter, was based on the shape of the distribution of first-day movements of recaptured marked moths released in the 1990 dispersal experiment (Brookes \& Butlin, 1994). 


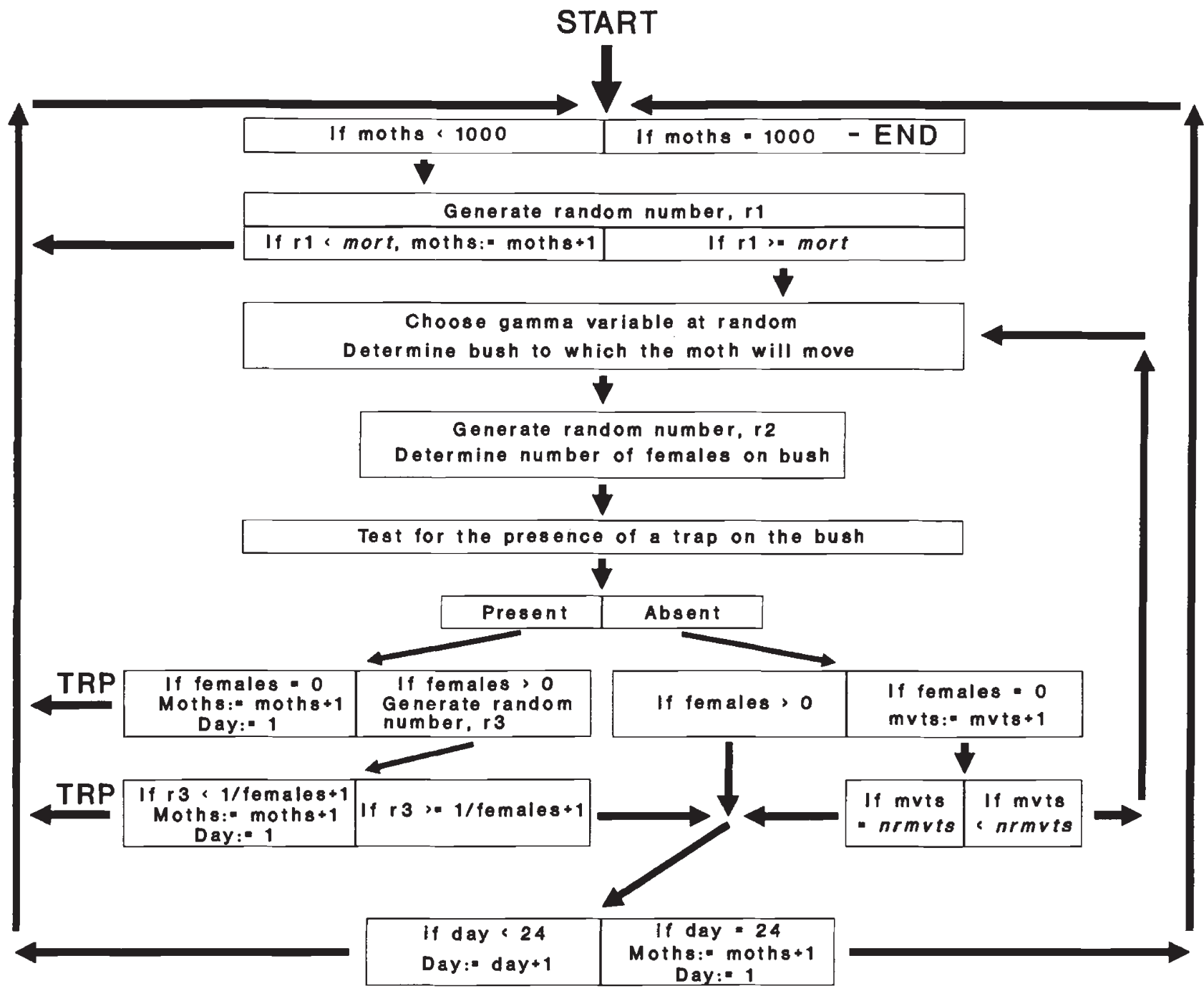

Fig. 1 Flow diagram representing the main body of the simulation program. At the start of the model, before the main body of the program is executed, the co-ordinates of all the bushes within the study population are entered, followed by the co-ordinates of the bushes with traps. Parameter values are then entered for gammafile, mort, nrmovements, maxfemales and sigmafemales.

The release bush co-ordinates and the corresponding observed data are also specified. Finally, the 'starting day' is entered. This determines the day on which the simulated release begins and is necessary to account for the different days on which some of the moths were released in the 1990 dispersal experiment (Brookes \& Butlin, 1993). The program ends when the movements of 1000 moths have been simulated. Abbreviations: ' $:=$ '= 'becomes equal to', 'TRP' = moth trapped, mvts = movements, $n r m v t s=n r m o v e m e n t s$. Words in italics refer to the specified model parameters. For further details see text.

Maxfemales and sigmafemales. The parameter maxfemales and its associated parameter sigmafemales are incorporated into the model to account for the fluctuations with time in the average number of wild calling females per bush. Field observations suggested that the presence of wild females affected the probability of male recapture. This effect was strongest about 10 days after the initial release of marked male moths into the study population, when the number of wild calling females reached its peak (Brookes \& Butlin, 1994). If a moth moves to a bush with a trap, the probability of the moth being caught in the trap is dependent on the number of wild calling females on the bush, which is determined from maxfemales and sigmafemales.

Nrmovements. The parameter nrmovements specifies the maximum number of potential inter bush movements which a male moth can make on any given day. Male moths have considerable flight potential and it seems plausible to assume that if a male moves to a bush which lacks either a trap or wild calling females, the male may move to another bush in search of a call- 
ing female. Obviously, there must be some physical limit to the number of inter bush movements an individual moth can make in a day, and this is set by the specified parameter.

\section{Calculation of goodness of fit}

The goodness of fit of a set of expected data to the observed data was tested using the natural logarithm of the likelihood function (the support function), which takes the form

$$
S(\theta)=\sum_{i=1}^{s} a_{i} \ln p_{i}(\theta)
$$

where $a_{i}=$ the observed number in the $i$ th class, $p_{i}(\theta)=$ the probability of the $i$ th class, in a total of $s$ classes (Edwards, 1972), and applying Yates' correction for continuity where necessary (Sokal \& Rohlf, 1969).

Although there is a total of $24 \times 45$ classes in the present study, some classes were combined to calculate the log likelihood function. The field data showed that most male moths were recaptured in traps $16 \mathrm{~m}$ or less from their release bush. Traps situated further from the release bushes rarely caught marked moths. Consequently, all the recaptures in the outer ring of traps surrounding a release bush (at approximately $32 \mathrm{~m}$ ) were combined over all days to form a single 'class'. A similar procedure was carried out for those moths marked at one release site but recaptured in the 30 traps surrounding the other two release bushes.

A final component of the total log likelihood function is concerned with the proportion of moths which is recaptured in all traps. A good fit of the expected data to the observed data depends not only on the proportions of expected recaptures in those traps in which moths were actually observed, but also on the total proportion of simulated moths which was recaptured compared with the observed data.

The optimal combinations of parameter values are those which correspond to peaks on the log likelihood surface of all possible parameter value combinations. The estimate of $\sigma$ is based on the combination of parameter values which overall best describe the recaptures of marked moths for all releases.

\section{Results}

Initially, seven values for the mortality parameter and three values for each of the other four parameters were tested in all possible combinations on each of the five releases, making a total of 2835 simulations. To assess how much of the total variance in the data could be attributed to the effect of each of the parameters, and whether there was any evidence of two-way interaction effects between parameters, the simulation data were analysed using ANOVA (GENSTAT 5), assuming that each parameter value represented a different treatment. $F$ ratios can therefore be used as a guide to interpreting the relative effects of each of the parameters on the variation observed in the log likelihood values (see Table 1).

Table 1 shows that individually each of the five parameters has a large effect, particularly sigmafemales and nrmovements. In comparison, the relative effects of the two-way interactions are very small, other than the nrmovements * sigmafemales interaction (see below). This indicates that the variation in the values of the observed treatment means (Table 2) primarily reflects the effect of the parameter value itself. Consequently, treatment means can be used to detect optimal values for each of the parameters. The observed trends in the log likelihood means for each of the parameter values indicated that the optimal values for some of the parameters may have been outside the range of values already tested (Table 2). For example, decreasing the value of gammafile produced smaller values of the mean log likelihood. With only three values tested for this parameter, it was impossible to detect whether the optimal value of gammafile was between 2.0 and 3.5 or whether it was actually less than 2.0. To increase the resolution of the log likelihood surface, it was necessary to run another series of simulations which included values either side of the values already tested. For this second series of simulations, two of the parameters held constant values. Nrmovements was kept at 1 and mort was kept at 0.25 . For the three values of

Table $1 F$ ratios for each of the five parameters and the two-way interactions between parameters

\begin{tabular}{lr}
\hline Source of variation & $F$ ratio \\
\hline mort & 235.21 \\
gammafile & 94.58 \\
maxfemales & 74.24 \\
sigmafemales & 457.81 \\
nrmovements & 308.99 \\
mort $*$ gammafile & 2.29 \\
mort $*$ maxfemales & 11.02 \\
mort $*$ sigmafemales & 19.85 \\
mort $*$ nrmovements & 10.35 \\
gammafile $*$ maxfemales & 2.77 \\
gammafile $*$ sigmafemales & 0.43 \\
gammafile $*$ nrmovements & 0.46 \\
maxfemales $*$ sigmafemales & 8.32 \\
maxfemales $*$ nrmovements & 17.46 \\
sigmafemales $*$ nrmovements & 72.74 \\
\hline
\end{tabular}


Table 2 Log likelihood means $\left(L_{\mathrm{m}}\right)$ for each of the parameter values tested in the initial set of simulations

\begin{tabular}{|c|c|c|c|c|c|c|c|}
\hline mort & 0.05 & 0.10 & 0.15 & 0.20 & 0.25 & 0.30 & 0.40 \\
\hline$L_{\mathrm{m}}$ & 91.99 & 83.27 & 79.78 & 77.91 & 77.20 & 77.04 & 77.17 \\
\hline gammafile & & 2.0 & & 3.5 & & 5.0 & \\
\hline$L_{\mathrm{m}}$ & & 78.37 & & 80.58 & & 82.92 & \\
\hline maxfemales & & 1.0 & & 3.0 & & 5.0 & \\
\hline$L_{\mathrm{m}}$ & & 82.95 & & 79.60 & & 79.32 & \\
\hline sigmafemales & & 3.0 & & 5.0 & & 7.0 & \\
\hline$L_{\mathrm{m}}$ & & 86.24 & & 79.02 & & 76.61 & \\
\hline nrmovements & & 1 & & 3 & & 5 & \\
\hline$L_{\mathrm{m}}$ & & 76.08 & & 81.68 & & 84.10 & \\
\hline
\end{tabular}

Means are in log likelihood units, minus signs have been removed.

nrmovements already tested, nrmovements $=1$ was found consistently to give the best fit to the observed data. Because a value of nrmovements less than 1 was meaningless in the context of the model, it was therefore reasonable to fix this value at 1 for all subsequent simulations.

Mort values of $0.25,0.30$ and 0.40 all gave very similar mean log likelihoods. A mort value of 0.25 was chosen both as a conservative estimate and because of evidence from field observations. Although most of the recaptured marked males were recaptured in the first week following their release, several males were recaptured up to 2 weeks after release (Brookes \& Butlin, 1994). With a constant daily mortality rate greater than 0.25 , it is highly improbable that such individuals would have been detected. The similarity in the mean $\log$ likelihoods for the two higher rates of mortality may be explained by the fact that the actual mortality rate in the field is not constant. Initially, during the time when most marked moths are caught, there may be high rates of mortality, possibly associated with the handling of the moths or because of density-dependent predation. After this time, mortality rates may decrease so that individuals that have survived may then persist in the population. Because only a few individuals were recaptured much later in the experiment, their effect on the overall log likelihood value is likely to be small. The higher mortality values may therefore be producing a slightly better fit to the observed data in the first week after release but the mort value of 0.25 compensates for this difference by accounting for the individuals caught later in the experiment. The mean log likelihood must eventually increase for values of mort greater than 0.40 because, at some stage, most moths will be suffering mortality before they are able to make any movements away from their release bush.

The results from the second series of simulations demonstrate that only variation in the values of gammafile and maxfemales have any appreciable effect
Table $3 F$ ratios for each of the three parameters tested individually and for the two- and three-way parameter interactions

\begin{tabular}{lr}
\hline Source of variation & $F$ ratio \\
\hline gammafile & 14.93 \\
maxfemales & 21.37 \\
sigmafemales & 0.26 \\
gammafile $*$ maxfemales & 0.35 \\
gammafile $*$ sigmafemales & 0.38 \\
maxfemales $*$ sigmafemales & 0.48 \\
gammafile $*$ maxfemales $*$ sigmafemales & 0.20 \\
\hline
\end{tabular}

Table 4 Log likelihood means $\left(L_{\mathrm{m}}\right)$ for each of the parameter values tested in the second set of simulations

\begin{tabular}{lccccc}
\hline gammafile & 1.0 & 1.5 & 2.0 & 2.5 & 3.0 \\
$L_{\mathrm{m}}$ & 77.13 & 73.64 & 72.85 & 72.95 & 73.24 \\
maxfemales & 0.5 & 1.0 & 3.0 & 5.0 & 7.0 \\
$L_{\mathrm{m}}$ & 72.30 & 72.18 & 73.15 & 74.91 & 77.27 \\
sigmafemales & 3.0 & 5.0 & 7.0 & 9.0 & 11.0 \\
$L_{\mathrm{m}}$ & 74.22 & 73.65 & 73.78 & 74.06 & 74.11 \\
\hline
\end{tabular}

Mort $=0.25$, nrmovements $=1$.

on the outcome of the mean log likelihood (Table 3 ). Neither sigmafemales nor any of the interactions had any effect. There was very little difference in the mean log likelihoods for maxfemales equal to $0.5,1.0$ or 3.0 (Table 4). The effect of this parameter is therefore primarily the result of the significant increases in the mean log likelihood for maxfemales equal to 5.0 and 7.0 , respectively. The optimal value of gammafile was found to be 2.0 although there were only slight increases in the mean log likelihood values at gammafile equal to 2.5 and 3.0 , respectively.

Therefore, in apparent contrast to the first series of simulations, sigmafemales had no effect on the out- 
come of the mean log likelihood and maxfemales only had an effect at unrealistically high values of the parameter. These differences are primarily a consequence of the large two-way interaction effect between nrmovements and sigmafemales (Table 1). The first series of simulations demonstrated that both nrmovements and sigmafemales had very large effects on the outcome of the mean log likelihoods. However, as outlined above, a value of nrmovements equal to 1 consistently gave the best overall fit to the observed data, no matter what the values of the other parameters. The very large effect observed for sigmafemales was primarily a manifestation of heterogeneity in the mean log likelihoods when nrmovements was greater than 1 .

\section{Estimates of $\sigma$}

The number of females on a bush on any given day, as determined by sigmafemales and maxfemales, therefore does not affect the movement of males to bushes (and hence traps); it only determines the probability of recapture once a male has landed on a trap bush. This result meant that when the traps were effectively removed from the model of dispersal, all the subroutines relating to the numbers of females on a bush could be removed from the program. This new simplified version of the model therefore assumes that males make a single daily interbush movement, as determined by gammafile. A male's dispersal is terminated when it dies, as determined by mort.

All subsequent simulations with the new version of the model were run with gammafile equal to 2.0 and mort equal to 0.25 . As in the previous model, for any particular combination of parameter values, the main body of the program is repeated 1000 times to simulate the dispersal of 1000 moths. Males make daily interbush movements until they die. The distance from the last bush a male landed on before it died to its original starting bush is recorded for each male. When the dispersal of all 1000 moths is completed, the axial variance of the 1000 distance measurements is calculated. The square root of this variance gives an estimate of the dispersal rate, $\sigma$.

Simulations were initiated in one of eight sample squares within the study population, each of side $50 \mathrm{~m}$, to examine how variation in bush density might affect the dispersal of males. Each moth begins its dispersal from a bush chosen at random from within the specified sample square.

The eight estimates of $\sigma$ shown in Table 5 are remarkably consistent and demonstrate that there is no effect of bush density on the extent of dispersal in male $Y$. padellus within the study population. When bush densities are low, the values of most of the variables picked at random from the chosen gamma distribution will be less than half the distance to the nearest bush. Consequently, most moths will tend to remain on the same bush for several days. Occasionally, a variable will be picked from the extreme tail of the distribution, enabling a moth to move the relatively large distance between bushes. Over the course of an entire simulation, these occasional long distance movements may produce an overall variance in dispersal distances equivalent to that for the more frequent, small-scale interbush movements made by moths in areas of high bush density.

It would be useful to know how much confidence to put on the estimates of $\sigma$ shown in Table 5. To an approximation, values of $\sigma$ which are more than two $\log$ likelihood units away from the observed value can be rejected (Edwards, 1972). For the purposes of the current model, gammafile values of 1.0, 2.0, 3.0 and 5.0 and mort values of $0.125,0.15,0.25$ and 0.50 were tested in all possible combinations. Although it is not possible to relate these values to specific confidence intervals, the extreme values of each of the parameters, particularly gammafile, almost certainly correspond to values of $\sigma$ which are some way outside the 95 per cent confidence interval of the observed value (see log likelihood means in Table 3). The use of these parameter combinations should therefore test how robust the estimates of $\sigma$ are, rather than putting specified confidence limits on the estimates.

Simulations using the parameter combinations described above were performed for moths 'randomly released' from sample squares 1 and 5, i.e. the squares with the highest and lowest bush densities, respectively (Table 6). As one might expect, decreasing the daily rate of mortality and increasing the value of gammafile

Table 5 Values of $\sigma$ for the simulated dispersal of 1000 moths in each of eight sample squares

\begin{tabular}{ccc}
\hline Sample square & $N$ & $\sigma(\mathrm{m})$ \\
\hline 1 & 35 & 3.9 \\
2 & 16 & 4.1 \\
3 & 18 & 4.4 \\
4 & 10 & 4.6 \\
5 & 6 & 4.0 \\
6 & 12 & 3.9 \\
7 & 15 & 4.1 \\
8 & 25 & 4.0 \\
& & Mean $=4.1$ \\
\hline
\end{tabular}

$N$ refers to the number of bushes within a sample square. $\sigma$ represents the axial standard deviation about the release points (equal along both axes). 
Table 6 Values of $\sigma$ for the simulated dispersal of 1000 moths according to the specifications of the model parameters shown

\begin{tabular}{|c|c|c|c|}
\hline Sample square & gammafile & mort & $\sigma(\mathrm{m})$ \\
\hline \multirow{4}{*}{1} & 1 & $\begin{array}{l}0.125 \\
0.25 \\
0.50\end{array}$ & $\begin{array}{l}2.3 \\
1.8 \\
1.4\end{array}$ \\
\hline & 2 & $\begin{array}{l}0.15 \\
0.25 \\
0.50\end{array}$ & $\begin{array}{l}4.2 \\
3.9^{*} \\
2.6\end{array}$ \\
\hline & 3 & $\begin{array}{l}0.15 \\
0.25 \\
0.50\end{array}$ & $\begin{array}{l}7.1 \\
6.2 \\
3.8\end{array}$ \\
\hline & 5 & $\begin{array}{l}0.15 \\
0.25 \\
0.50\end{array}$ & $\begin{array}{r}11.8 \\
9.8 \\
7.0\end{array}$ \\
\hline \multirow{4}{*}{5} & 1 & $\begin{array}{l}0.125 \\
0.25 \\
0.50\end{array}$ & $\begin{array}{l}1.7 \\
1.5 \\
1.2\end{array}$ \\
\hline & 2 & $\begin{array}{l}0.15 \\
0.25 \\
0.50\end{array}$ & $\begin{array}{l}5.2 \\
4.0^{*} \\
2.8\end{array}$ \\
\hline & 3 & $\begin{array}{l}0.15 \\
0.25 \\
0.50\end{array}$ & $\begin{array}{l}8.8 \\
6.7 \\
4.2\end{array}$ \\
\hline & 5 & $\begin{array}{l}0.15 \\
0.25 \\
0.50\end{array}$ & $\begin{array}{r}14.8 \\
11.9 \\
7.4\end{array}$ \\
\hline
\end{tabular}

Simulations were initiated in sample squares 1 and 5, respectively.

$*=$ 'best' estimate (as shown in Table 5).

both have the effect of increasing $\sigma$. For the extreme values of each parameter, $\sigma$ tends to be larger for moths whose dispersal was initiated in the low density sample square. As parameter values become more extreme, the interbush distances become less important determinants of the actual distances moved. As interbush distances will be greater in areas of low bush density, individual movements by moths will tend to be larger. The maximum estimate of $\sigma$ was $14.8 \mathrm{~m}$ in sample square 5, with gammafile equal to 5.0 and mort equal to 0.15 . However, as outlined above, this value probably represents a loss of more than two log likelihood units from the observed value and is therefore an unrealistically high upper estimate. A value of about $9 \mathrm{~m}$ is probably a more realistic estimate of the upper confidence limit.
All the estimates shown in Table 6 are well within the same order of magnitude and this suggests that the model is quite robust, even to quite large deviations in the value of parameters away from their optimal values. The mean estimate of $\sigma$ of $4.1 \mathrm{~m}$ for the optimal values of gammafile, nrmovements and mort (2.0, 1 and 0.25 , respectively; Table 5) would appear to be a relatively confident estimate; $\sigma$ is probably not more than $9 \mathrm{~m}$ and will certainly not exceed $15 \mathrm{~m}$.

\section{Discussion}

The aim of this study was to develop and run a simulation model of male dispersal for Y. padellus, using field observations of actual recapture data as its basis, to obtain an accurate estimate of the dispersal rate for male moths within the study population. Using this approach, the distorting effect of traps can effectively be removed from an estimate of the dispersal rate. Although the model was based on a specific population with its own, possibly unique, set of biological parameters, there is no reason why the general approach presented could not be applied to other species which share some of the dispersal characteristics evident in $Y$. padellus.

The estimates of $\sigma$ assume that all of a male's genes will be transported to the last bush on which it lands before it dies. As males can potentially reproduce at any time during their adult lifetime, the estimate of $\sigma$ is likely to be an overestimate of the actual rate at which genes spread within the population. If a male has a uniform probability of mating on any given day then, on average, each male will mate halfway through its adult life. Assuming that there is no covariance between the $x$ and $y$ components of movement (i.e. the variance in distance moved increases linearly with time), then the actual variance of gene flow distances will be half the total lifetime variance. Therefore, a more realistic estimate of $\sigma$ is $\sqrt{ }\left(4.1^{2} / 2\right)=2.9 \mathrm{~m}$. An approximate upper confidence limit of $\sigma=\sqrt{ }\left(9^{2}\right)$ $2)=6.4 \mathrm{~m}$ can be put on this estimate. If most mating is concentrated towards the beginning of a male's life then this estimate is likely to be further reduced.

The locations at which reproduction in $Y$. padellus takes place are ultimately determined by the locations of the appropriate host bushes. However, variation in bush density had little or no effect on the eventual outcome of the dispersal rate (Table 5) and hence the rate of gene flow. Ultimately, the rate of gene flow was determined by the movement parameter, gammafile. Although the parameter is obviously critical to the success of the model, variation in the value of gammafile around its optimal value of 2.0 had little effect on the dispersal rate. We can therefore be relatively confident in the final estimate of $\sigma$. 
What are the implications of these results for the population structure of $Y$. padellus? Firstly, any gene frequency fluctuations produced by genetic drift will manifest themselves over geographical scales of the order of 3-6 m. Within the study population, the average distance between a bush and its nearest neighbour is $5.4 \mathrm{~m}$ (Brookes \& Butlin, 1994). This suggests that the dispersal range of most males will be confined to the bushes immediately adjacent to those on which they emerged as adults. The amplitude of any gene frequency fluctuations will depend on the effective population size within this dispersal range, i.e. the neighbourhood size $\left(N_{b}\right)$. Wright (1951) has shown that $N_{b}$ must be relatively small for drift to produce differentiation of neighbourhoods. If $N_{b}$ is 1000 or more, neighbourhoods behave as if under a state of universal panmixia. However, there is likely to be some differentiation if $N_{b}$ is of the order of 200 and appreciable differentiation if $N_{b}$ is of the order of 20 or less. Estimates of the neighbourhood size for $Y$. padellus within the study population range from a minimum of two individuals to a maximum of 164 individuals (Brookes \& Butlin, 1994), and are small enough to suggest that genetic drift could have important effects on the genetic differentiation of populations. Local fluctuations in the gene frequencies of neighbourhoods may result in the differentiation of much larger areas (Wright, 1951). There will be a correlation among the gene frequencies of adjacent neighbourhoods which will decrease as the distance between neighbourhoods increases. As dispersal is apparently so restricted in $Y$. padellus, these genetic correlations may break down over relatively small geographical scales.

These results question the putative existence of genetically differentiated host races in $Y$. padellus (Menken, 1981, 1989; Menken et al., 1992). A large component of the genetic differentiation observed between host-associated populations of $Y$. padellus may be a general manifestation of the fine-scale population structure of this species. Host-associated polymorphism must be detected on both local (i.e. within the dispersal range) and broader geographical scales to support the existence of host races (Feder et al., 1990).

\section{Acknowledgements}

The work was funded by an SERC studentship to M.B.

\section{References}

BROOKES, M. I. AND BUTLIN, R. K. 1994. Population structure in the small ermine moth Yponomeuta padellus: an estimate of male dispersal. Ecol. Entomol. (in press).

EDWARDS, A. W. F. 1972. Likelihood. Cambridge University Press, London.

ENDLER, J. A. 1979. Gene flow and life history patterns. Genetics, 93, 263-284.

FEDER, J. L., CHILCOTE, C. A. AND BUSH, G. L. 1990. Regional, local and microgeographic allele frequency variation between apple and hawthorn populations of Rhagoletis pomonella in western Michigan. Evolution, 44, 595-608.

MENKEN, S. B. J. 1981. Host races and sympatric speciation in small ermine moths, Yponomeutidae. Entomol. Exp. Appl., 30, 280-292.

MENKEN, S. B. J. 1989. Electrophoretic studies on geographic populations, host races, and sibling species of insect pests. In: Loxdale, H. D. and Den Hollander, J. (eds) Electrophoretic Studies on Agricultural Pests, pp. 181-202. Clarendon Press, Oxford.

MENKEN, S. B. J., HERREBOUT, w. M. AND WIEBES, J. T. 1992. Small ermine moths (Yponomeuta): their host relations and evolution. Ann. Rev. Entomol., 37, 41-66.

MOOD, A. M., GRAYBILL, F. A. AND BOES, D. C. 1974. Introduction to the Theory of Statistics. McGraw-Hill, New York.

ROHLF, F. J. AND SCHNELL, D. 1971. An investigation of the isolation-by-distance model. Am. Nat., 105, 295-324.

Slatkin, M. 1985. Gene flow in natural populations. Ann. Rev. Ecol. Syst., 16, 393-430.

SOKAL, R. R. AND ROHLF, F. J. 1969. Biometry. W. H. Freeman, San Francisco.

WRIGHT, s. 1943. Isolation by distance. Genetics, 28, 114-138.

WRIGHT, S. 1951. The genetical structure of populations. Ann. Eugen., 15, 323-354. 